Sci. USA 93, 5319-5324 (1996).

10. Kiyosue, T. et al. Control of fertilization-independent endosperm development by the MEDEA polycomb gene in Arabidopsis. Proc. Natl Acad. Sci. USA 96, 4186-4191 (1999).

11. Luo, M. et al. Genes controlling fertilization-independent seed development in Arabidopsis thaliana. Proc. Natl Acad. Sci. USA 96, 296-301 (1999).

12. Köhler, C. et al. Arabidopsis MSIl is a component of the MEA/FIE Polycomb group complex and required for seed development. EMBO J. 22, 4804-4814 (2003).

13. Lu, X. \& Horvitz, H. R. lin-35 and lin-53, two genes that antagonize a C. elegans Ras pathway, encode proteins similar to $\mathrm{Rb}$ and its binding protein RbAp48. Cell 95, 981-991 (1998).

14. Dahiya, A., Wong, S., Gonzalo, S., Gavin, M. \& Dean, D. C. Linking the Rb and polycomb pathways. Mol. Cell 8, 557-569 (2001)

15. Ach, R. A., Taranto, P. \& Gruissem, W. A conserved family of WD- 40 proteins binds to the retinoblastoma protein in both plants and animals. Plant Cell 9, 1595-1606 (1997).

16. Weinberg, R. A. The retinoblastoma protein and cell cycle control. Cell 81, 323-330 (1995)

17. Harbour, J. W. \& Dean, D. C. The Rb/E2F pathway: expanding roles and emerging paradigms. Genes Dev. 14, 2393-2409 (2000).

18. Ach, R. A. et al. RRB1 and RRB2 encode maize retinoblastoma-related proteins that interact with a plant D-type cyclin and geminivirus replication protein. Mol. Cell. Biol. 17, 5077-5086 (1997).

19. De Veylder, L. et al. Control of proliferation, endoreduplication and differentiation by the Arabidopsis E2Fa-DPa transcription factor. EMBO J. 21, 1360-1368 (2002)

20. Lee, E. Y. et al. Mice deficient for $\mathrm{Rb}$ are nonviable and show defects in neurogenesis and haematopoiesis. Nature 359, 288-294 (1992).

21. Jacks, T. et al. Effects of an Rb mutation in the mouse. Nature 359, 295-300 (1992).

22. Clarke, A. R. et al. Requirement for a functional Rb-1 gene in murine development. Nature 359, 328-330 (1992).

23. Kwee, H. S. \& Sundaresan, V. The NOMEGA gene required for female gametophyte developmen encodes the putative APC6/CDC16 component of the Anaphase Promoting Complex in Arabidopsis. Plant J. 36, 853-866 (2003).

24. Du, W. \& Dyson, N. The role of RBF in the introduction of G1 regulation during Drosophila embryogenesis. EMBO J. 18, 916-925 (1999).

25. Honys, D. \& Twell, D. Comparative analysis of the Arabidopsis pollen transcriptome. Plant Physiol. 132, 640-652 (2003).

26. Yan, W., Kero, J., Suominen, J. \& Toppari, J. Differential expression and regulation of the retinoblastoma family of proteins during testicular development and spermatogenesis: roles in the control of germ cell proliferation, differentiation and apoptosis. Oncogene 20, 1343-1356 (2001).

27. Bracken, A. P. et al. EZH2 is downstream of the pRB-E2F pathway, essential for proliferation and amplified in cancer. $E M B O$ J. 22, 5323-5335 (2003).

28. Li, Y., Rosso, M. G., Strizhov, N., Viehoever, P. \& Weisshaar, B. GABI-Kat SimpleSearch: a flanking sequence tag (FST) database for the identification of T-DNA insertion mutants in Arabidopsis thaliana. Bioinformatics 19, 1441-1442 (2003).

29. Vielle-Calzada, J. P. et al. Maintenance of genomic imprinting at the Arabidopsis medea locus requires zygotic DDM1 activity. Genes Dev. 13, 2971-2982 (1999).

30. Alexander, M. P. Differential staining of aborted and nonaborted pollen. Stain Technol. 44, 117-122 (1969).

Acknowledgements We are grateful to M. Collinge, J. Fütterer, U. Grossniklaus, M. Hanin and L. Hennig for discussions and critical reading of the manuscript. We thank J. Wyrzykowska and A. Johnston for help with in situ hybridization and ovule clearings. We also thank the Salk Institute Genomic Analysis Laboratory for providing the sequence-indexed Arabidopsis T-DNA insertion mutants and $\mathrm{ABRC}$ for providing us with seeds. This work was supported by funding from the Swiss National Science Foundation to W.G.

Competing interests statement The authors declare that they have no competing financial interests.

Correspondence and requests for materials should be addressed to W.G (wilhelm.gruissem@ipw.biol.ethz.ch).

\section{Structural basis of protein phosphatase 1 regulation}

\section{Mohammed Terrak*, Frederic Kerff*, Knut Langsetmo, Terence Tao \& Roberto Dominguez}

Boston Biomedical Research Institute, 64 Grove Street, Watertown, Massachusetts 02472, USA

* These authors contributed equally to this work

The coordinated and reciprocal action of serine/threonine (Ser/Thr) protein kinases and phosphatases produces transient phosphorylation, a fundamental regulatory mechanism for many biological processes. The human genome encodes a far greater number of Ser/Thr protein kinases than of phosphatases. Protein phosphatase 1 (PP1), in particular, is ubiquitously distributed and regulates a broad range of cellular functions, including glycogen metabolism, cell-cycle progression and muscle relaxation $^{1,2}$. PP1 has evolved effective catalytic machinery but lacks substrate specificity. Substrate specificity is conferred upon PP1 through interactions with a large number of regulatory subunits. The regulatory subunits are generally unrelated, but most possess the RVxF motif, a canonical PP1-binding sequence. Here we reveal the crystal structure at $2.7 \mathrm{~A}$ resolution of the complex between PP1 and a 34-kDa N-terminal domain of the myosin phosphatase targeting subunit MYPT1. MYPT1 is the protein that regulates PP1 function in smooth muscle relaxation ${ }^{3}$. Structural elements amino- and carboxy-terminal to the RVxF motif of MYPT1 are positioned in a way that leads to a pronounced reshaping of the catalytic cleft of PP1, contributing to the
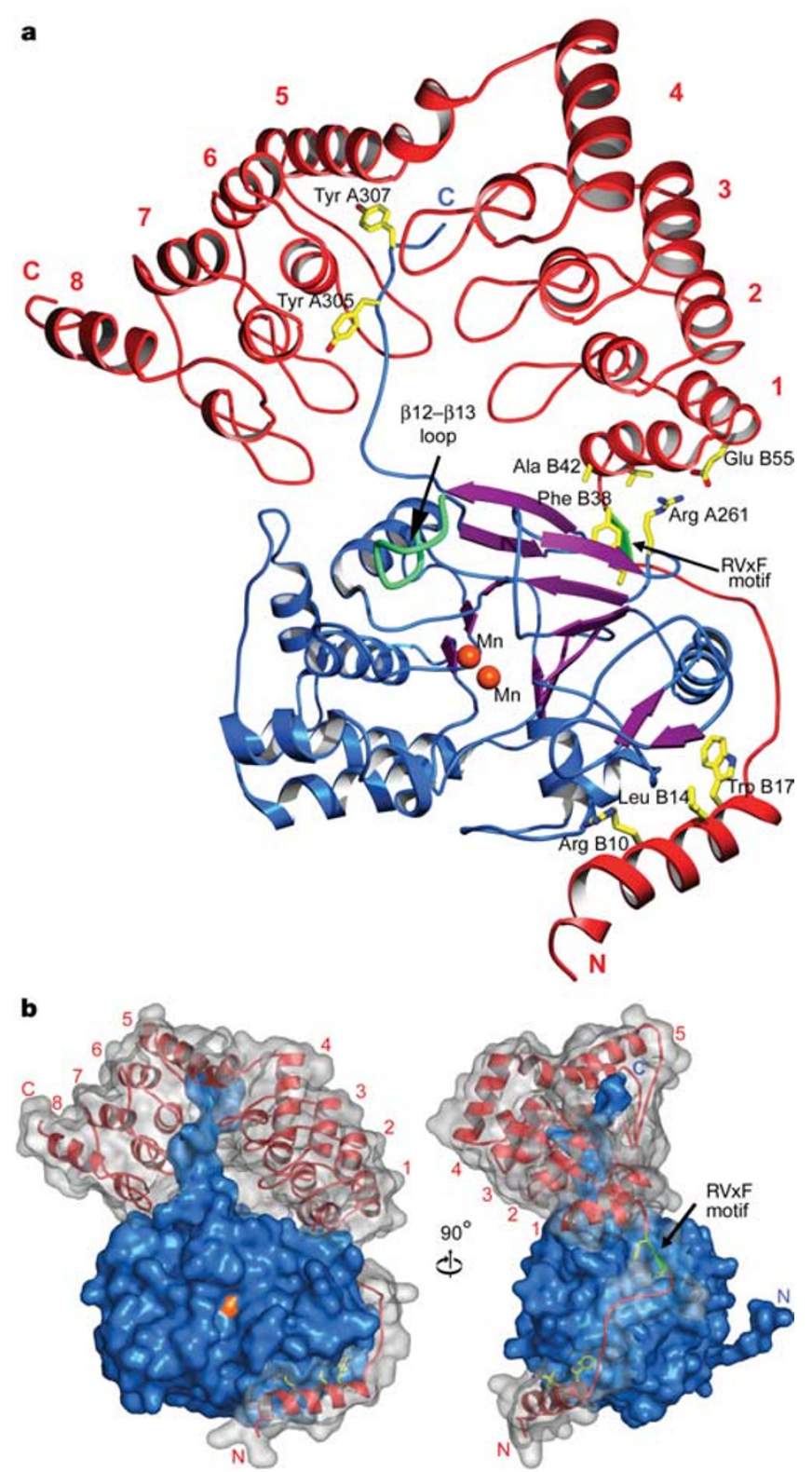

Figure 1 General fold of the PP1 $\delta-\mathrm{MYPT}_{1-299}$ complex. a, Ribbon representation: PP1 $\delta$; $\alpha$-helices and loops (blue) and $\beta$-strands (magenta); and MYPT1 (red). The two cations in the catalytic site are coloured orange. The ankyrin repeats of MYPT1 are numbered 1 to 8 from the $\mathrm{N}$ to the $\mathrm{C}$ terminus. The major contacts with PP1 involve three separate regions of MYPT1: the N-terminal arm, the RVxF motif and the second group of ankyrin repeats, which interact mainly with PP1 residues Tyr A305 and Tyr A307. b, Two surface representations of the complex, rotated by 90 degrees (PP1 $\delta$, blue; MYPT1, red). 
increased myosin specificity of this complex. The structure has general implications for the control of PP1 activity by other regulatory subunits.

In smooth muscle and non-muscle cells, phosphorylation of Ser 19 of the myosin regulatory light chain (RLC) turns on the ATPase activity of myosin. Myosin light chain kinase (MLCK) and myosin phosphatase catalyse the reactions of phosphorylation and dephosphorylation, regulating muscle contraction and relaxation, respectively. Myosin phosphatase is a trimeric holoenzyme, comprising a catalytic subunit (PP1, $\delta$-isoform), a $130-\mathrm{kDa}$ targeting subunit (MYPT1) and a $20-\mathrm{kDa}$ subunit (M20) of unknown function $^{3}$. MYPT1 plays a key part in determining the physical and functional integrity of the trimeric myosin phosphatase holoenzyme. It binds PP1 $\delta$ at the $\mathrm{N}$ terminus ${ }^{4-8}$ and M20 at the $\mathrm{C}$ terminus $^{3,4}$. Binding to myosin seems to take place through two separate sites: a site situated within the C-terminal one-third of MYPT1 (ref. 9), and a site responsible for catalysis that engages PP1 and the N-terminal one-third of MYPT1 simultaneously. In a similar way to that of the intact myosin phosphatase holoenzyme, the complex between PP1 and the N-terminal one-third of MYPT1 displays $\sim 15$-fold increased catalytic activity and $\sim 10$-fold higher affinity for phosphorylated myosin (or P-RLC) than the isolated catalytic subunit ${ }^{4-7,10}$. In addition, this complex has reduced activity towards other substrates, such as phosphorylase $a$ and glycogen synthase ${ }^{4,5,7}$. Together these findings suggest that different parts of MYPT1 are responsible for substrate specificity and targeting.

Here we reveal the $2.7-\AA$-resolution structure of the complex between chicken gizzard PP1 $\delta$ and amino acids Met 1 to Ser 299 of MYPT1 (MYPT1 $1_{1-299}$ ) (see Methods). Crystal structures have been determined previously for complexes of PP1 ( $\alpha$ and $\gamma$ isoforms) with three natural toxin inhibitors: microcystin $^{11}$, okadaic acid ${ }^{12}$ and calyculin A (ref.13), with tungstate ${ }^{14}$ and a peptide from the muscle glycogen-targeting regulatory subunit $\left(G_{M}\right)^{15}$. Despite the differences in PP1 isoforms, crystallization conditions and crystal packing contacts, these structures are very similar to one another and to the structure obtained here for PP1 $\delta-M Y P T 1_{1-299}$. The overall fold of PP1 consists of two tightly linked domains, an N-terminal $\alpha / \beta$ domain (amino acids A1-A160; chain A designates PP1 $\delta$ ) and a C-terminal $\beta$ domain (A161-A327), which also contains three $\alpha$-helices. Most of the $\beta$-strands from the two domains converge at the inter-domain interface, where they form a $\beta$-sandwich made of two mixed $\beta$-sheets (Fig. 1). The catalytic site lies within a large Y-shaped cleft at the inter-domain interface ${ }^{11-13}$. The three branches of the Y-shaped cleft are commonly referred to as the hydrophobic, acidic and C-terminal grooves (Fig. 2a). Separating the acidic and the $\mathrm{C}$-terminal grooves is a protruding loop (Asn 271 to Asp 277), which connects $\beta$-strands $\beta 12$ and $\beta 13$. This loop is ideally positioned to interact with PP1 substrates and inhibitors ${ }^{11-13}$ and has been linked with inhibitor specificity ${ }^{16}$. There are two metal ions in the catalytic site of the PP1 $1-\mathrm{MYPT}_{1-299}$ structure. By analogy with previous structures ${ }^{11-13}$, and because of the presence of $\mathrm{Mn}^{2+}$ in the purification and crystallization buffers, we have identified these ions as $\mathrm{Mn}^{2+}$. Note that the addition of $\mathrm{Mn}^{2+}$ is required for full activity of this complex. However, we cannot rule out that one of the two cations might be $\mathrm{Fe}^{2+}$, as in the PP1 $\gamma$-tungstate structure ${ }^{14}$.

The most noticeable differences between the current and previous structures of PP1 (refs 11-15) occur at the $\mathrm{N}$ - and C-terminal ends, where the sequences of PP1 isoforms diverge the most. Previous structures of PP1 $\alpha$ (ref. 11) and PP1 $\gamma$ (refs 12-15) typically begin with Leu 7 and end at Ala 299 (Ser 299 in PP1ס); that is, 25 to 30 amino acids are disordered at the $\mathrm{C}$ termini of these structures. In the current structure Glu A300 to Gly A309 are trapped between the two groups of ankyrin repeats of MYPT1 and, as a result, are ordered. Thus, this structure suggests that some regulatory subunits may interact with the divergent and flexible $\mathrm{C}$ termini of PP1 to mediate isoform specificity.

MYPT1 interacts with PP1 over a large $\left(2,560 \AA^{2}\right)$ intermolecular interface. This interaction, involving primarily the C-terminal $\beta$-domain of PP1, does not lead to any noticeable structural change in PP1, other than the partial stabilization of the C-terminal end. The absence of an allosteric effect may be unexpected, yet MYPT1 has a profound effect on the global shape and charge distribution of the catalytic cleft of PP1 (Fig. 2). To understand this point better we divide MYPT1 conceptually into three functional domains: the $\mathrm{N}$-terminal arm (amino acids B1-B34; chain B designates MYPT1), the RVxF motif (amino acids B35-B38) and the ankyrin repeat domain (amino acids B39-B299).

The $34 \mathrm{~N}$-terminal amino acids of MYPT1 preceding the RVxF motif form a long arm that wraps around PP1 to reach the base of the Y-shaped catalytic cleft (Figs 1 and 2). Part of this arm folds into an $\alpha$-helix (Met B3 to Gly B19). Hydrophobic interactions involving a

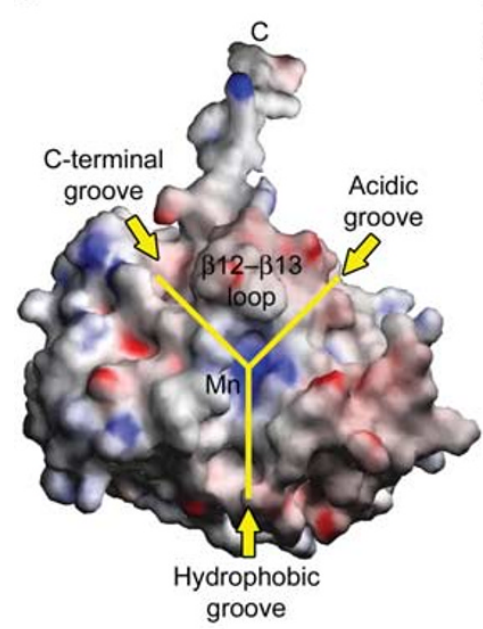

b

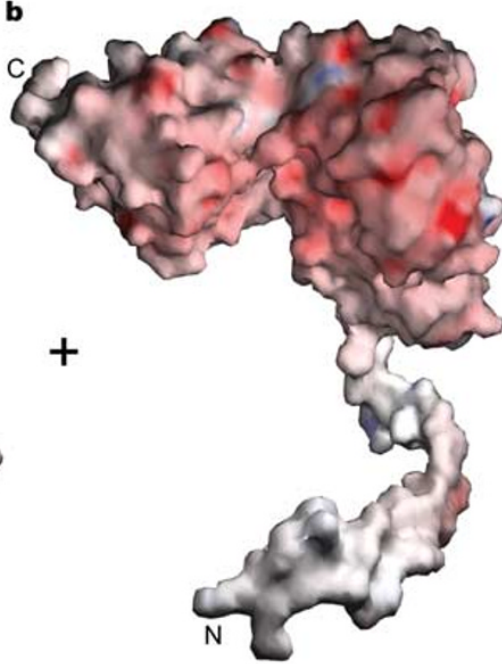

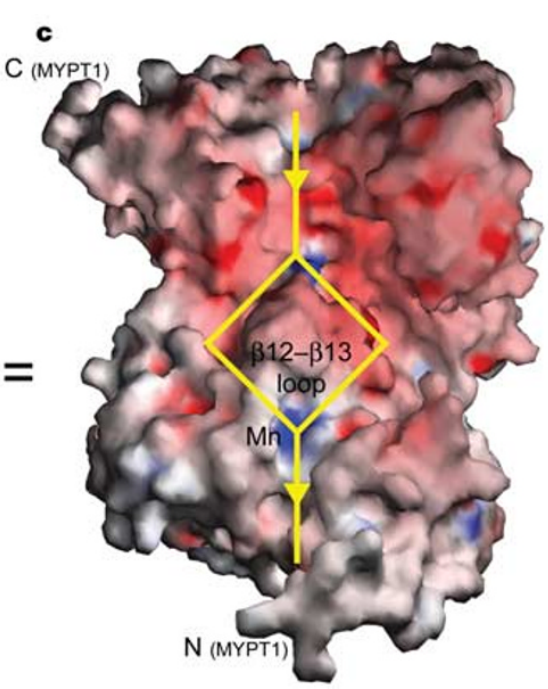

d

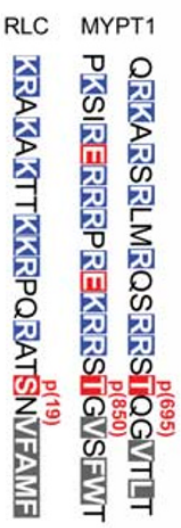

Figure 2 Electrostatic surface representations of the PP1 $1-\mathrm{MYPT}_{1-299}$ complex. a-c, Surfaces of PP1 (a), MYPT1 (b) and their complex (c) calculated using identical parameters (red and blue indicate regions charged negatively and positively, respectively). The Y-shaped catalytic cleft of PP1 is composed of three grooves: hydrophobic, acidic and C-terminal. The $\beta 12-\beta 13$ loop separates the acidic and the C-terminal grooves. The reshaping of the catalytic cleft of PP1 results from the binding of the N terminus of MYPT1 near the hydrophobic groove and the addition of the acidic cleft of the ankyrin repeats at the other end of the cleft. d, Sequences of the RLC around Ser 19 and the MYPT1 around the regulatory phosphorylation sites Thr 695 and Thr 850. Note the existing charge complementarity between these sequences and the catalytic cleft of myosin phosphatase. 
residues in this $\alpha$-helix and a hydrophobic pocket in PP1 determine the position of the N-terminal arm (Fig. 3a).

The location of the $\mathrm{N}$ terminus of MYPT1 in the structure fits the biochemical data well. A peptide corresponding to the sequence Met B1 to Phe B38 of MYPT1 can confer upon PP1 some of the myosin specificity and increased activity characteristic of the myosin phosphatase holoenzyme $e^{4,6-8}$. However, a shorter peptide (Asp B23 to Phe B38) binds to PP1 but has no effect on its catalytic activity $^{7}$. Together these results emphasize the importance of the $\mathrm{N}$ terminus of MYPT1 for the myosin specificity of myosin phosphatase. Whereas the structure negates the existence of an allosteric effect on PP1, it places the N terminus of MYPT1 near the hydrophobic groove of the catalytic cleft, where it may interact with the myosin substrate directly (steric effect). Hydrophobic residues at the N terminus of MYPT1 (Met B1, Met B3 and Ala B6) and the side chain of Lys B2, add to the general hydrophobicity of this branch of the catalytic cleft of PP1 (Fig. 2). Hydrophobic amino acids C-terminal to Ser 19 of the myosin RLC are likely to interact within this part of the cleft, making contacts with the $\mathrm{N}$ terminus of MYPT1 (Fig. 2d).

The numerous PP1 regulatory subunits share no significant sequence similarity, but most possess the RVxF motif, a specific PP1 targeting sequence ${ }^{2}$. The crystal structure of $\mathrm{PP} 1 \gamma$ complexed with a $R V x F$-containing peptide from the $G_{M}$ subunit reveals six amino acids $\left({ }^{63}\right.$ RRVSFA $\left.^{69}\right)$ bound in a hydrophobic pocket distal to the catalytic site ${ }^{15}$. In what seems to be a similar manner, the side chains of the Val and Phe residues in the RVxF motif of MYPT1 $\left({ }^{35} \mathrm{KVKF}^{38}\right)$ are embedded in the same pocket in PP1 $($ Fig. $3 \mathrm{c}, \mathrm{d}$ ). Residues Val B36 to Asp B39 adopt an extended conformation and are incorporated as an additional $\beta$-strand to a $\beta$-sheet in PP1, parallel to the $\mathrm{N}$-terminal half of $\beta 14$. Also making up part of this cluster of interactions are the side chains of Ala B42 and Val B43 from the first ankyrin repeat of MYPT1. Finally, Asp A242 of PP1 makes two hydrogen-bonding contacts with the main-chain nitro-

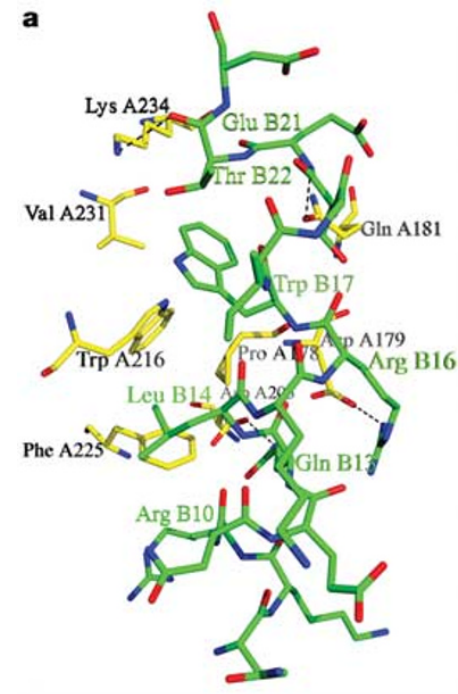

c

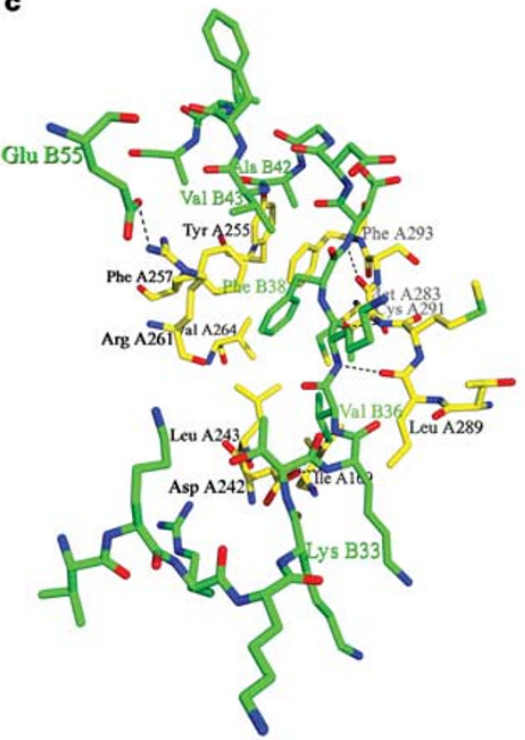

Figure $\mathbf{3}$ Interactions between PP1 and MYPT1. a, Stereo diagram of the interactions between the N-terminal arm of MYPT1 (green carbon atoms) and PP1 (yellow carbon atoms). b. The same region of MYPT1 superimposed onto an electrostatic surface representation of PP1, where red, blue and white indicate negatively-charged, positivelycharged and non-charged regions, respectively. c, Stereo diagram of the interactions
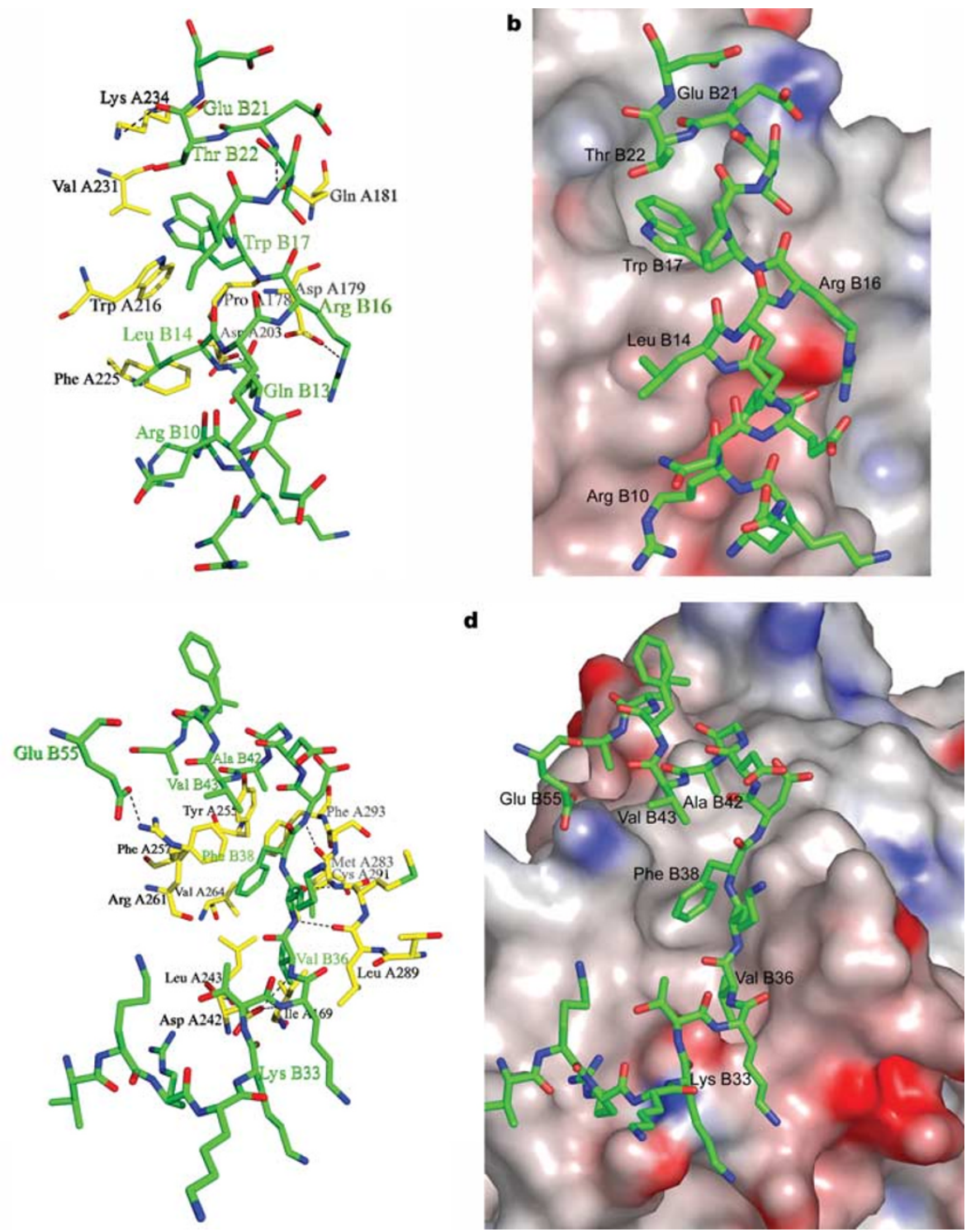

involving the RVxF motif and the first $\alpha$-helix of ankyrin repeat 1. $\mathbf{d}$, The same region of MYPT1 superimposed onto an electrostatic surface representation of PP1. Note that the basic sequence preceding the RVxF motif does not interact directly with the acidic patch at the lower right corner of this figure. 
gen atoms of Lys B35 and Val B36 of MYPT1. The ensemble of these interactions represents the single most important contribution to the formation of the PP1-MYPT1 complex. Other interactions observed in this structure are comparatively weaker and can be expected to be specific for this complex. As such, these interactions are primarily relevant to the modulation of PP1 activity.

In MYPT1, as in other PP1 regulatory subunits, positively charged amino acids at the $\mathrm{N}$ terminus $\left({ }^{30} \mathrm{KRKK}^{33}\right)$ and negatively charged amino acids at the $\mathrm{C}$ terminus $\left({ }^{39} \mathrm{DD}^{40}\right)$ flank the $\mathrm{RVxF}$ motif. It has been suggested ${ }^{15}$ that the basic region preceding the $\mathrm{RVxF}$ motif may interact with a negatively charged patch near the RVxF-binding pocket (Fig. 3d). We do not observe such an interaction. The amino acids $\mathrm{N}$-terminal to the $\mathrm{RVxF}$ motif in MYPT1 follow a path away from the negatively charged patch, making only a few interactions with PP1, mostly through mainchain atoms.

Amino acids B39-B291 of MYPT1 fold into two groups of four ankyrin repeats, separated by a hinge at Glu B172 (Fig. 1 and Supplementary Fig. 1). Ankyrin repeats are $\sim 33$-amino-acid-long motifs consisting of pairs of antiparallel $\alpha$-helices connected by $\beta$-hairpin loops ${ }^{17}$. In MYPT1, repeats 1, 2, 3, 6 and 7 are canonical with the sole exception that repeat 1 forms an extra helical turn at the $\mathrm{N}$ terminus. Repeats 4 and 8 lack the $\beta$-hairpin loop and repeat 5 lacks the first $\alpha$-helix. The interactions with PP1 are limited to repeats 1, 5, 6 and 7 (Fig. 1). Repeat 1 interacts with the RVxFbinding pocket through hydrophobic amino acids located within the extra helical turn at the $\mathrm{N}$ terminus (Fig. 3c). A salt bridge between Glu B55 and PP1 residue Arg A261 also involves repeat 1. The remaining interactions are almost exclusively between the second group of ankyrin repeats and the C terminus of PP1, involving residues Tyr A305 and Tyr A307 in particular. Because sequence variations between PP1 isoforms cluster towards the $\mathrm{C}$ termini, a possible outcome of this interaction is to determine PP1 isoform specificity. Indeed, $\mathrm{PP} 1 \gamma$ lacks both Tyr residues. In PP $1 \alpha$, only Tyr A305 is present, although shifted by one amino acid.

The ankyrin repeat is a widespread motif, generally associated with a protein-protein interaction function ${ }^{17}$. The present structure offers an interesting twist: the ankyrin repeats of MYPT1 seem to play only a limited part in binding, but a potentially key part in modulating the catalytic activity of PP1. This finding is in agreement with biochemical evidence that shows almost no binding to PP1 of MYPT1 constructs lacking the N-terminal 38 amino acids ${ }^{4-8}$. Constructs, however, that add the ankyrin repeat domain to the $\mathrm{N}$ terminus of MYPT1 display increased myosin specificity and reduced activity towards other substrates, comparable to full-length myosin phosphatase $e^{4-6,8}$.

Substrate binding to the isolated ankyrin repeat domain is also weak ${ }^{5}$. This may reflect the fact that binding to myosin depends on a three-dimensional organization that can only exist when MYPT1 binds to PP1. Indeed, the $\beta$-hairpin loops from the two groups of ankyrin repeats roughly face each other in the structure, forming a clamp-like structure that closes around the C terminus of PP1. This organization gives rise to a large acidic cleft on one side of the interface between the two groups of ankyrin repeats. In the structure, this cleft is positioned as an extension to the catalytic cleft of PP1 (Fig. 2). The catalytically important $\beta 12-\beta 13$ loop of $\mathrm{PP} 1^{16}$ forms a protrusion right in the centre of this enlarged cleft.

The major outcome from the formation of the PP1-MYPT1 complex is to produce an extended catalytic cleft specifically adapted for the myosin substrate and, at the same time, less compatible with other substrates. Analysis of the sequence of the myosin RLC around the important phosphorylation site Ser 19 makes this point fully apparent (Fig. 2d). Fifty per cent of the sequence N-terminal to Ser 19 is charged positively and can be expected to bind within the expanded acidic groove of PP1 $\delta-$ MYPT1. The sequence C-terminal to Ser 19 is mainly hydrophobic and probably binds at the hydrophobic groove, which has also been adapted for this function by the proximity of the $\mathrm{N}$ terminus of MYPT1. This hypothesis assumes that the directionality of the polypeptide chain of the bound substrate is from the acidic to the hydrophobic groove. This turns out to be the directionality of the auto inhibitory peptide of the structurally related phosphatase PP2B (calcineurin) ${ }^{18}$. The sequences of two regulatory phosphorylation sites in MYPT1 (Thr 695 and Thr 850) resemble that of the RLC around Ser 19. Therefore, these sequences are also good candidates to bind at the catalytic cleft of PP1 $\delta$-MYPT1 (Fig. 2d). Whereas phosphorylation of Thr 695 inhibits myosin phosphatase $^{19}$, that of Thr 850 has so far been linked with an effect on myosin binding but not on the catalytic activity'.

Among the regulatory subunits of PP1, p53BP2 is the only one where ankyrin repeats follow the RVxF motif, as in MYPT1. Because p53BP2 binds with higher affinity to PP1 than to the tumour suppressor p53 (ref. 20) and inhibition of PP1 is linked to phosphorylation of p53 and apoptosis ${ }^{21}$, it is possible that p53BP2 provides a structural link between PP1 and p53. This interaction can now be modelled by superimposing the ankyrin repeats of p53BP2, from the structure of its complex with p53 (ref. 22), onto those of MYPT1.

Analysis of the sequences of various regulatory subunits reveals important inhibitory/modulatory sequences $\sim 34$ amino acids $\mathrm{N}$-terminal or $\sim 22$ amino acids C-terminal to the $\mathrm{RVxF}$ motif. Because 34 amino acids $\mathrm{N}$-terminal to the RVxF motif allow MYPT1 to reach the catalytic cleft of PP1, other regulatory subunits may follow a similar path, including the nuclear inhibitor of PP1 (NIPP1) ${ }^{23}$, spinophilin and neurabin ${ }^{24}$ (Fig. 4). Amino acids 143-217 of NIPP1 constitute a potent PP1 inhibitor ${ }^{23}$. This region contains regulatory phosphorylation sites at Ser 178, Ser 199,
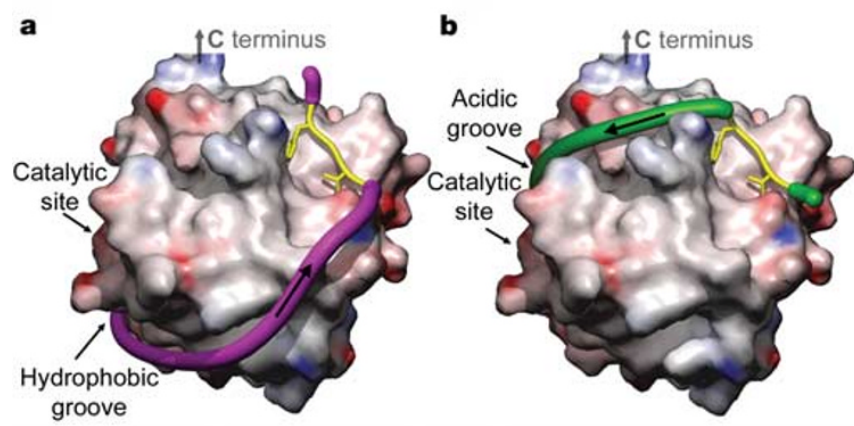

c

MYPT1 MKMADAKQKRNEQLKRWI GSETDLEPPV ${ }^{28}$ NIPP1 156 LPEEETELDNLTEFNTAHNKRISTLTIEEGNLDI QRPK 193 Neurabin ${ }^{413}$ SDWGETGTEQDEEEDSDEINSYYQPDMEYSE I VGLPEEE ${ }^{40}$ Spinophilin 404 DSAGSALEEDDEEDEEDGEPPYEPESGCVE I PGLSEEE 439 Inhibitor-1 PP-32

MYPT NIPP1 194 RKRKNSRVTFSEDDEI I NPEDVDPSVGRFRNMVQTAVV 231 Neurabin ${ }^{451}$ E I PANRK I KFSSAP I KVFNTYSNEDYDRRNDEVDPVAA 480 Spinophilin 440 DPAPSRK I HFSTAPI QVFSTYSNEDYDRRNEDVDPMAA 477 Inhibitor-1 3 QDNSPRKI QFTVPLLEPHLDPEAAEQI/RRRRPTPATLV 40

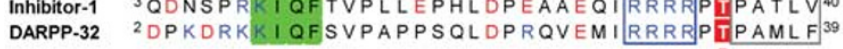

Figure 4 Model illustrating two possible paths between the RVxF motif and the catalytic site of PP1. a, Path N-terminal to the RVxF motif (magenta) suggested by the structure of PP1-MYPT1. NIPP1, neurabin and spinophilin may follow this path. b, Path C-terminal to the RVxF motif (green) leading to the cleft by way of the acidic groove. Inhibitor-1 and DARPP-32 may follow this path in order to position the inhibitory P-Thr 35 at the catalytic site, allowing for favourable electrostatic interactions between the basic sequence $\mathrm{N}$-terminal to P-Thr 35 and the acidic groove of PP1. c, Sequences of some PP1 regulatory subunits around the RVxF motif (shaded green). Acidic and basic sequences that may potentially interact with PP1 are outlined in red and blue, respectively. Inhibitory phosphorylation sites are shaded red. 
Thr 161 and Ser 204, and the consensus RVxF motif, which is preceded by a basic sequence as in MYPT1. A highly acidic sequence (EEETELD) 35 amino acids N-terminal to the RVxF motif coincides with one of the phosphorylation sites. This sequence could possibly target the highly basic metal cluster in the catalytic site of PP1. Also consistent with the current structure is an alternative path that leads to the catalytic cleft C-terminal to the RVxF motif (Fig. 4). As previously proposed ${ }^{25}$, inhibitor 1 and the dopamine and cAMPregulated phosphoprotein DARPP-32 may follow this second path.

The structure of PP1-MYPT1 presented here reveals how the binding of a regulatory subunit adapts the catalytic cleft of PP1 to perform a specific function. In this case, that function is the dephosphorylation of myosin that leads to smooth muscle relaxation. The interactions involving the $\mathrm{RVxF}$ motif play an important part in the formation of the complex. However, other weaker interactions modulate PP1 activity and isoform specificity. We predict that features revealed by this structure will apply to other PP1 regulatory subunits.

\section{Methods}

\section{Protein preparation}

The gene encoding chicken gizzard PP1 (UniProt P37140) was inserted into the pTYB12 IMPACT expression vector from New England BioLabs that leads to expression of the fusion protein chitin-binding domain-intein-PPl $\delta$. The gene encoding MYPT1 $1_{1-299}$ (UniProt Q90624) was inserted into the expression plasmid pACYC184, which is compatible with pTYB12, and placed under the control of a T7 promoter. The two vectors were introduced into Escherichia coli strain BL21 (DE3) for co-expression. The complex PP $1 \delta-\mathrm{MYPT}_{1-299}$ was purified using a chitin affinity column. Because expression levels were higher for MYPT1 $1_{1-299}$ than for PP1 $\delta$, the affinity tag was added to the latter, to ensure that a 1:1 complex was obtained during purification. After elution from the chitin column, following dithiothreitol-induced self-cleavage of the intein, the complex was loaded into a MonoQ column (Pharmacia) and eluted using a 150 to $400 \mathrm{mM}$ gradient of $\mathrm{NaCl}$ in $20 \mathrm{mM}$ Tris- $\mathrm{HCl}$ buffer $\mathrm{pH} 7.5,1 \mathrm{mM} \mathrm{MnCl}, 2 \mathrm{mM}$ dithiothreitol.

\section{Crystallization}

Before crystallization the PP1 $\delta-\mathrm{MYPT}_{1-299}$ complex was dialysed overnight against $10 \mathrm{mM}$ Tris buffer $\mathrm{pH} 7.5,20 \mathrm{mM} \mathrm{NaCl}, 4 \mathrm{mM}$ dithiothreitol and $1 \mathrm{mM} \mathrm{MnCl}_{2}$ and concentrated to $\sim 8 \mathrm{mg} \mathrm{ml}^{-1}$. The complex was crystallized at $20^{\circ} \mathrm{C}$ using the hangingdrop vapour diffusion method by mixing $2 \mu \mathrm{l}$ of protein solution and $2 \mu \mathrm{l}$ of a precipitant solution, containing 9.2\% (w/v) poly(ethylene glycol) (PEG) 5,000 monomethylether, $35 \%$ (v/v) glycerol and $200 \mathrm{mM} \mathrm{NH}_{4} \mathrm{Cl}$.

\section{Data collection, structure determination and refinement}

A data set (to $2.7 \AA$ resolution) was collected at $100 \mathrm{~K}$ at BioCARS beamline 14-BM-C from a crystal of PP1 $\delta-M Y P T 1_{1-299}$. The diffraction data were indexed and scaled using the programs DENZO and SCALEPACK ${ }^{26}$ (Supplementary Table 1). A molecular replacement solution for the PP1 $1 \delta$ part of the structure was obtained using the program $\mathrm{AMoRe}^{27}$ and the structure of PPl $\alpha$ (Protein Data Bank code 1FJM) as a search model. After screening a number of ankyrin-repeat-containing structures, a molecular replacement solution was found for a group of three repeats derived from the structure of P18-Ink4C (Protein Data Bank code 1IHB). An electron-density map was calculated with phases derived from the partial model resulting from the molecular replacement solutions of PP1 and the three ankyrin repeats. The map revealed the location of another group of three ankyrin repeats. Additional refinement led to the identification of two additional, non-canonical repeats (repeats 4 and 8). Refinement and model-building were done using the programs $\mathrm{CNS}^{28}$ and $\mathrm{O}^{29}$. The quality of the refined structure was validated with the programs PROCHECK ${ }^{30}$ and CNS. The final structure includes PP1 $\delta$ amino acids A1-A309 (chain A), MYPT1 amino acids B1-B291 (chain B), two $\mathrm{Mn}^{2+}$ ions, a molecule of PEG 5,000 monomethylether and 228 water molecules. Notice that PP1 residue His A1 is the last of three amino acids left over by the intein construct (the other two being disordered in the structure). The PP1 $\delta$ chain was numbered starting from this residue to preserve the same notation as for PP1 $\alpha$ and PPl $\gamma$, which have one additional amino acid at their $\mathrm{N}$ termini.

Received 30 January; accepted 16 April 2004; doi:10.1038/nature02582.

Published online 26 May 2004

1. Bollen, M. Combinatorial control of protein phosphatase-1. Trends Biochem. Sci. 26, 426-431 (2001).
2. Cohen, P. T. Protein phosphatase-1-targeted in many directions. J. Cell Sci. 115, 241-256 (2002).

3. Hartshorne, D. J., Ito, M. \& Erdodi, F. Myosin light chain phosphatase: subunit composition, interactions and regulation. J. Muscle Res. Cell Motil. 19, 325-341 (1998).

4. Johnson, D. F. et al. Identification of protein-phosphatase-1-binding domains on the glycogen and myofibrillar targeting subunits. Eur. J. Biochem. 239, 317-325 (1996).

5. Hirano, K., Phan, B. C. \& Hartshorne, D. J. Interactions of the subunits of smooth muscle myosin phosphatase. J. Biol. Chem. 272, 3683-3688 (1997).

6. Tanaka, J. et al. Interaction of myosin phosphatase target subunit 1 with the catalytic subunit of type 1 protein phosphatase. Biochemistry 37, 16697-16703 (1998).

7. Toth, A. et al. Study of the subunit interactions in myosin phosphatase by surface plasmon resonance. Eur. J. Biochem. 267, 1687-1697 (2000).

8. Gailly, P. et al. Regions of the 110-kDa regulatory subunit M110 required for regulation of myosinlight-chain-phosphatase activity in smooth muscle. Eur. J. Biochem. 239, 326-332 (1996).

9. Velasco, G., Armstrong, C., Morrice, N., Frame, S. \& Cohen, P. Phosphorylation of the regulatory subunit of smooth muscle protein phosphatase $1 \mathrm{M}$ at Thr 850 induces its dissociation from myosin. FEBS Lett. 527, 101-104 (2002).

10. Ichikawa, K. et al. Interactions and properties of smooth muscle myosin phosphatase. Biochemistry 35 , 6313-6320 (1996).

11. Goldberg, J. et al. Three-dimensional structure of the catalytic subunit of protein serine/threonine phosphatase-1. Nature 376, 745-753 (1995).

12. Maynes, J. T. et al. Crystal structure of the tumor-promoter okadaic acid bound to protein phosphatase-1. J. Biol. Chem. 276, 44078-44082 (2001).

13. Kita, A. et al. Crystal structure of the complex between calyculin A and the catalytic subunit of protein phosphatase 1. Structure 10, 715-724 (2002).

14. Egloff, M. P., Cohen, P. T., Reinemer, P. \& Barford, D. Crystal structure of the catalytic subunit of human protein phosphatase 1 and its complex with tungstate. J. Mol. Biol. 254, 942-959 (1995).

15. Egloff, M. P. et al. Structural basis for the recognition of regulatory subunits by the catalytic subunit of protein phosphatase 1. EMBO J. 16, 1876-1887 (1997).

16. Connor, J. H., Kleeman, T., Barik, S., Honkanen, R. E. \& Shenolikar, S. Importance of the beta12 betal3 loop in protein phosphatase- 1 catalytic subunit for inhibition by toxins and mammalian protein inhibitors. J. Biol. Chem. 274, 22366-22372 (1999).

17. Sedgwick, S. G. \& Smerdon, S. J. The ankyrin repeat: a diversity of interactions on a common structural framework. Trends Biochem. Sci. 24, 311-316 (1999).

18. Kissinger, C. R. et al. Crystal structures of human calcineurin and the human FKBP12-FK506calcineurin complex. Nature 378, 641-644 (1995).

19. Feng, J. et al. Inhibitory phosphorylation site for Rho-associated kinase on smooth muscle myosin phosphatase. J. Biol. Chem. 274, 37385-37390 (1999).

20. Helps, N. R., Barker, H. M., Elledge, S. J. \& Cohen, P. T. Protein phosphatase 1 interacts with p53BP2, a protein which binds to the tumour suppressor p53. FEBS Lett. 377, 295-300 (1995).

21. Long, X., Wu, G., Gaa, S. T. \& Rogers, T. B. Inhibition of protein phosphatase-1 is linked to phosphorylation of p53 and apoptosis. Apoptosis 7, 31-39 (2002).

22. Gorina, S. \& Pavletich, N. P. Structure of the p53 tumor suppressor bound to the ankyrin and $\mathrm{SH} 3$ domains of 53BP2. Science 274, 1001-1005 (1996).

23. Beullens, M. et al. Molecular determinants of nuclear protein phosphatase-1 regulation by NIPP-1. J. Biol. Chem. 274, 14053-14061 (1999).

24. Terry-Lorenzo, R. T. et al. The neuronal actin-binding proteins, neurabin I and neurabin II, recruit specific isoforms of protein phosphatase-1 catalytic subunits. J. Biol. Chem. 277, 27716-27724 (2002).

25. Barford, D., Das, A. K. \& Egloff, M. P. The structure and mechanism of protein phosphatases: insights into catalysis and regulation. Annu. Rev. Biophys. Biomol. Struct. 27, 133-164 (1998).

26. Otwinowski, Z. \& Minor, W. Processing of X-ray diffraction data collected in oscillation mode. Methods Enzymol. 276, 307-326 (1997).

27. Navaza, J. AMoRe: an automated package for molecular replacement. Acta Crystallogr. A 50, 157-163 (1994)

28. Brunger, A. T. et al. Crystallography \& NMR system: A new software suite for macromolecular structure determination. Acta Crystallogr. D 54, 905-921 (1998).

29. Jones, T. A., Zou, J. Y., Cowan, S. W. \& Kjeldgaard, M. Improved methods for building protein models in electron density maps and location of errors in these models. Acta Crystallogr. A 47, 110-119 (1991).

30. Laskowski, R. A., MacArthur, M. W., Moss, D. S. \& Thornton, J. M. PROCHECK: a program to check the stereochemical quality of protein structures. J. Appl. Crystallogr. 26, 283-291 (1993).

\section{Supplementary Information accompanies the paper on www.nature.com/nature.}

Acknowledgements R.D. and T.T. were supported by NIH grants. Use of the Advanced Photon Source was supported by the US Department of Energy. The NIH supported use of the BioCARS facilities. We thank B.-J. Gong for assistance with protein expression during the early stages of this work, T. Kitazawa for discussions and A. Borus for reading the manuscript.

Competing interests statement The authors declare that they have no competing financial interests.

Correspondence and requests for materials should be addressed to R.D. (dominguez@bbri.org). Coordinates have been deposited in the Protein Data Bank under accession number 1S70. 